\title{
Minimum Spectral Bandwidth in Echo Seeded Free Electron Lasers
}

\author{
Erik Hemsing * \\ SLAC National Accelerator Laboratory, Menlo Park, CA, United States
}

This paper examines the impact of non-linear longitudinal phase distortions on the spectral bandwidth in echo seeded free electron lasers (FELs). It extends the existing theory developed in Hemsing [1] for echo-enabled harmonic generation (EEHG) to include finite laser pulse durations. An analytic expression for the shape of the optimized longitudinal bunching envelope is derived, and is used to determine the laser and electron beam pulse durations that minimize the seeded bandwidth in the presence of arbitrary phase distortions. The time-bandwidth product (TBP) is also derived, and is shown that the TBP and the bandwidth increase by no more than $\sqrt{2}$ from their transform-limited values when the bandwidth is minimized.

Keywords: FEL (free electron laser), EEHG, echo, seeded, xray, chirp, nonlinear, harmonic

\section{OPEN ACCESS}

Edited by:

Arya Fallahi,

Foundation for Research on

Information Technologies in Society,

ETH Zurich, Switzerland

Reviewed by:

Ilia L. Rasskazov,

University of Rochester, United States

Masoud Mehrjoo,

Helmholtz Association of German

Research Centers (HZ), Germany

${ }^{*}$ Correspondence:

Erik Hemsing

ehemsing@slac.stanford.edu

Specialty section:

This article was submitted to Optics and Photonics,

a section of the journal

Frontiers in Physics

Received: 03 October 2018

Accepted: 26 February 2019

Published: 22 March 2019

Citation:

Hemsing E (2019) Minimum Spectral

Bandwidth in Echo Seeded Free

Electron Lasers. Front. Phys. 7:35.

doi: 10.3389/fphy.2019.00035

\section{INTRODUCTION}

Externally seeded FELs use lasers to produce coherent high harmonic density modulations (bunching) in relativistic electron beams that are then used for the emission of coherent radiation at short wavelengths. Seeding is useful for overcoming the otherwise noisy output of SASE (self-amplified spontaneous emission) from these beams to generate narrowband FEL radiation. Besides improved longitudinal coherence, external seeding also enables control over the character of the FEL output pulses [2,3], allowing adjustable coherent bandwidths [4] or the ability to produce multiple, phase locked pulses [5, 6], or multiple colors $[7,8]$.

Several different external seeding schemes have been proposed, and a few demonstrated (see e.g., [9] and references therein). Relevant specifically to this work, echo-enabled harmonic generation (EEHG) is a scheme that uses two external laser pulses (generally UV wavelengths) to efficiently generate bunching in the electron beam down to soft $\mathrm{x}$-rays $[10,11]$. In EEHG, the first laser produces a sinusoidal energy modulation in the electron beam that is typically only a few times the intrinsic slice energy spread. The beam then goes through a strong longitudinally dispersive section that folds over the sinusoids and creates a filamentary phase space structure in the particle distribution. The second laser then modulates the filamented phase space distribution, and a final dispersive section creates high harmonic bunching at short wavelengths in the current distribution. The beam then enters the FEL where coherent light is emitted and exponentially amplified.

A primary goal of seeding schemes is to minimize the FEL bandwidth and produce transform-limited pulses. In principle, the narrowest obtainable bandwidth is given by the inverse electron beam bunch length. In practice however, electron beams in modern FELs have distortions in the longitudinal phase space that mix additional frequencies into the harmonic up-conversion that spoil the purity of the final output spectrum [12-20]. In Hemsing [1] a general method was developed to calculate the impact of beam energy distortions through the broadening they introduce in the bunching spectrum (i.e., the Fourier transform of the current distribution). The broadening width was then used to set limits on the amplitude of common distortions to maintain near transform-limited seeding. 
The magnitude, shape, and location of these distortions, within both the electron beam and the laser pulse, is an important factor in the optimization of external seeding schemes. The analysis in Hemsing [1] assumed infinitely long lasers that completely covered the electron beam (e-beam). In this limit, the bunching spectrum is predicted to be highly sensitive to e-beam energy variations because the whole beam contributes to the final spectrum and can have strong nonlinear portions in the head and tail. However, in practice, the seed lasers can be comparable in length to the electron beam or shorter, and positioned longitudinally within the e-beam to avoid seeding in the strong nonlinear portions. Shorter pulses naturally have broader bandwidths, so in order to achieve the narrowest harmonic bunching spectrum, a balance must be found between the transform limited bandwidth of the short laser and the broadening from the nonlinear phase contributions.

Here, using an extension of the formalism in Hemsing [1], we examine this more practically relevant case where the seed laser pulse can vary in duration, and also carry nonlinear temporal phase structures. This allows analysis of the bunching spectrum in the presence of nonlinearity in the electron beam and laser phase. From a general expression for the bandwidth, we show that laser pulses optimized in duration can produce much narrower bunching spectra once the e-beam energy distortions reach a threshold value. We also define the requirements that allow the laser phase to precisely compensate the e-beam distortions to obtain transform limited pulses.

The paper is arranged as follows. We first present an extension to the theory in Hemsing [1] that includes a finite seed laser pulse that produces a modulation with a longitudinal profile given by the function $g(z)$. Under reasonable approximations, the bunching bandwidth can be calculated straightforwardly from $g(z)$, the e-beam distribution $f(z)$, and the combined phase distortions $\varphi(z)$. Since the spectral-temporal properties in EEHG are inherited primarily from the second laser, we assume the first laser is infinite. Assuming that the EEHG bunching amplitude is optimized, we then derive an analytic expression for $g(z)$ with a Gaussian seed laser. From this we retrieve the well-known $m^{-1 / 3}$ harmonic compression effect, and are able to calculate the transform-limited bandwidth. Arbitrary phase distortions are then included as a Taylor series, and their impacts to each order on the total bandwidth and time-bandwidth product (TBP) are then calculated analytically. We then derive the conditions on the laser pulse length and e-beam length to minimize the bandwidth, and study a few examples.

\section{MATHEMATICAL DESCRIPTION}

Notation closely follows that of Xiang [11]. Consider an EEHG electron beam transformation of the form,

$$
\begin{aligned}
p_{1} & =p+A_{1} \sin \left(k_{1} z\right)+\Delta p_{1}(z) \\
z_{1} & =z+B_{1} p_{1} / k_{1} \\
p_{2} & =p_{1}+A_{2}\left(z_{1}\right) \sin \left[k_{2} z_{1}+\psi_{2}\left(z_{1}\right)\right]+\Delta p_{2}\left(z_{1}\right), \\
z_{2} & =z_{1}+B_{2} p_{2} / k_{1} .
\end{aligned}
$$

where the normalized laser modulations are $A_{1,2}=\Delta E_{1,2} / \sigma_{E}$, normalized dispersions are $B_{1,2}=k_{1} R_{56}^{(1,2)} \sigma_{E} / E$, the slice energy spread is $\sigma_{E}$, and $E$ is the electron beam energy. Here, distinct from the analysis in Hemsing [1], we allow the second laser to have a finite pulse length, $A_{2}\left(z_{1}\right)$, as well as a longitudinally dependent phase $\psi_{2}\left(z_{1}\right)$. The first laser, $A_{1}$, is assumed to be ideal and infinite in length. Additional energy distortions in the electron beam, $\Delta p_{1}$ and $\Delta p_{2}$, are again modeled as occurring alongside the laser modulations.

The bunching spectrum near the harmonic peak $k_{E}=a_{E} k_{1}=$ $(n+m K) k_{1}$ is given by

$$
\begin{aligned}
b_{n, m}(k)= & e^{-\xi_{E}^{2} / 2} J_{n}\left(-\xi_{E} A_{1}\right) \int e^{-i z\left(k-k_{E}\right)+i \varphi(z)} \\
& \times f(z) J_{m}\left(-a_{E} A_{2}(z) B_{2}\right) d z,
\end{aligned}
$$

where $\xi_{E}=n B_{1}+a_{E} B_{2}$ is the EEHG scaling parameter, and $f(z)$ is the longitudinal electron beam distribution function. The beam is assumed to have an uncorrelated Gaussian initial energy distribution. Similar to Hemsing [1], here we utilize the weaklyfolding approximation which assumes that the energy structures satisfy $B_{1} d \Delta p_{1} / d z \ll k_{1}$ and are small enough so that they do not lead to large changes in the phase space distribution after the first chicane. We also assume that the longitudinal variations in $\Delta p_{2}$, $A_{2}$, and $\psi_{2}$ are sufficiently slowly-varying that the full integral can be simplified by replacing $z$ for $z_{1}$ in the function arguments. It is also assumed that the system is far from the minimum pulse duration limit [21].

With these approximations, the phase $\varphi(z)$ captures the combined impact of energy distortions in the electron beam, $\Delta p_{1,2}$, and the phase variation $\psi_{2}$ in the second laser,

$$
\varphi(z)=-\xi_{E} \Delta p_{1}(z)-a_{E} B_{2} \Delta p_{2}(z)+m \psi_{2}(z) .
$$

The $z$-dependence of the second laser envelope $A_{2}(z)$ is still within the argument of the $J_{m}$ Bessel function, which complicates simple analytic solutions. We therefore search for an ansatz of the form,

$$
J_{m}\left(-a_{E} A_{2}(z) B_{2}\right)=g(z) J_{m}\left(-a_{E} A_{2} B_{2}\right),
$$

where the imprint of the slowly-varying laser envelope is captured by the function $g(z)$, determined shortly by approximate expansion. Note that in the limit of an infinitely long laser, $g \rightarrow 1$. In anticipation of a Gaussian laser profile, $A_{2}=A_{2}(0)$ is the peak of the modulation.

With $b_{n, m}(k)=\int \tilde{b}_{n, m}(z) e^{-i k z} d z$, the longitudinal bunching factor can then be expressed as

$$
\tilde{b}_{n, m}(z) \approx \bar{b}_{n, m} f(z) g(z) e^{i k_{E} z+i \varphi(z)},
$$

where $f(z) g(z)$ together form the $z$-dependent envelope, and $\bar{b}_{n, m}=e^{-\xi_{E}^{2} / 2} J_{n}\left(-\xi_{E} A_{1}\right) J_{m}\left(-a_{E} A_{2} B_{2}\right)$ the optimized bunching amplitude [20]. Now the instantaneous spatial bunching frequency is just the $z$-derivative of the full longitudinal phase,

$$
k_{z}(z)=k_{E}+\varphi^{\prime}(z)
$$


The average frequency is then

$$
\left\langle k_{z}\right\rangle=\frac{\int_{-\infty}^{\infty} k_{z}(z)[f(z) g(z)]^{2} d z}{\int_{-\infty}^{\infty}[f(z) g(z)]^{2} d z}=k_{E}+\left\langle\varphi^{\prime}(z)\right\rangle,
$$

and the spectral bandwidth of the harmonic bunching spike is

$$
\begin{aligned}
\sigma_{k}^{2} & =\sigma_{k_{E}}^{2}+\left\langle\left(\varphi^{\prime}\right)^{2}\right\rangle-\left\langle\varphi^{\prime}\right\rangle^{2}, \\
& =\sigma_{k_{E}}^{2}+\sigma_{\varphi^{\prime}}^{2},
\end{aligned}
$$

where the transform-limited bunching bandwidth is [22]

$$
\sigma_{k_{E}}^{2}=\frac{\int\left[(f g)^{\prime}\right]^{2} d z}{\int(f g)^{2} d z}
$$

This gives the bandwidth due to the combination of the laser modulation $g(z)$ with the electron beam distribution function $f(z)$. It is dominated by the shorter of the two if they coincide longitudinally. The bandwidth due to the nonlinear phase structures is given by $\sigma_{\varphi^{\prime}}$.

\section{OPTIMIZED MODULATION}

We consider the effect of a Gaussian laser pulse in the second echo modulator on the longitudinal profile of the bunching. This will enable us to calculate an approximate form for $g(z)$, and thus the harmonic compression factor and scaling of the bunching bandwidth in the presence of the phase $\varphi(z)$. Slippage in the modulator is ignored.

The optimum amplitude of the second laser from timeindependent echo theory is $A_{2}=j_{m, 1}^{\prime} / a_{E} B_{2}$, given by the requirement that $J_{m}\left(a_{E} A_{2} B_{2}\right)$ is peaked, with $j_{m, 1}^{\prime}=m+$ $0.8087 m^{1 / 3}$ the first zero of the Bessel function $J_{m}^{\prime}$. Small relative changes in the bunching due to variations from the optimum modulation are given by [20]

$$
\frac{\Delta \bar{b}_{n, m}}{\bar{b}_{n, m}}=-\left(\frac{\Delta A_{2}}{A_{2}}\right)^{2} \frac{\left(j_{m, 1}^{\prime}\right)^{2}-m^{2}}{2},
$$

where $m \gg 1$ is the harmonic of the second laser. Let us assume that the laser modulation envelope has an rms intensity duration $\sigma_{L} / c$,

$$
A_{2}(z)=A_{2} e^{-z^{2} / 4 \sigma_{L}^{2}}
$$

To lowest order near the $z=0$ peak, small changes in the laser amplitude vary according to $\Delta A_{2} / A_{2}=-z^{2} / 4 \sigma_{L}^{2}$. This suggests that the bunching envelope function $g(z)$ can be written as $g(z) \approx 1-\left(\frac{z^{2}}{4 \sigma_{L}^{2}}\right)^{2} \frac{\left(j_{m, 1}^{\prime}\right)^{2}-m^{2}}{2}$. Mathematically, this looks like a lowest order expansion about $z=0$ for small values of the second term. We posit then, that the functional dependance of $g(z)$ may be modeled as a super-Gaussian distribution that has the same series expansion to lowest order:

$$
\begin{aligned}
g(z) & \approx \exp \left[-\left(\frac{z^{2}}{4 \sigma_{L}^{2}}\right)^{2} \frac{\left(j_{m, 1}^{\prime}\right)^{2}-m^{2}}{2}\right] \\
& =\exp \left[-\frac{q^{2}}{2}\left(\frac{z}{\sigma_{g}}\right)^{4}\right]
\end{aligned}
$$
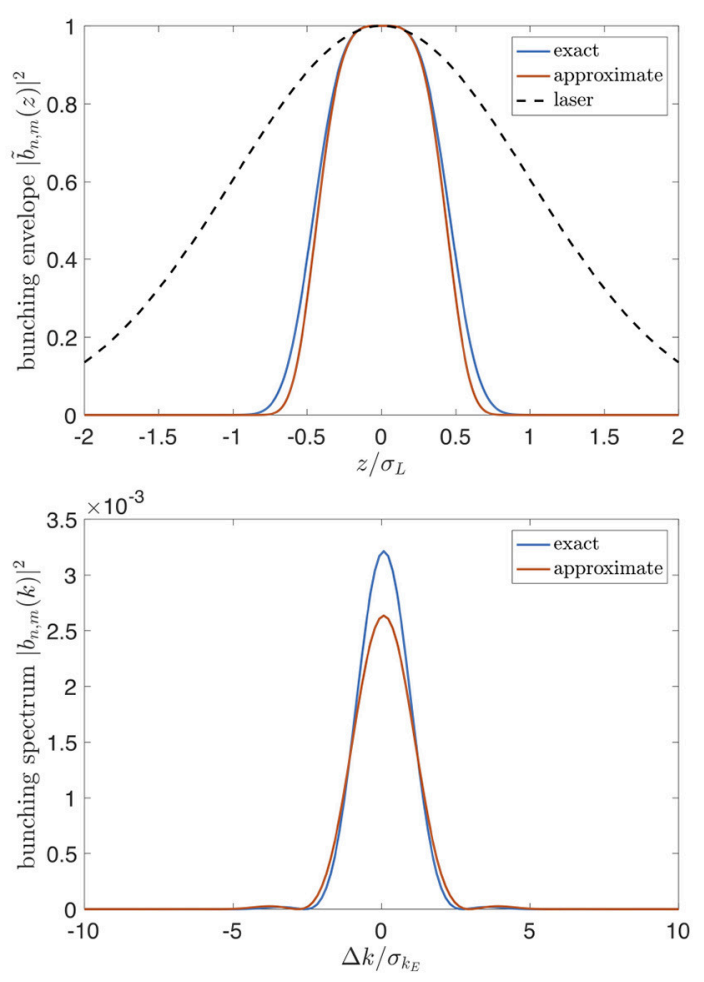

FIGURE 1 | (Top) Bunching envelope and harmonic compression effect for $m=50$. The approximate super-Gaussian $g(z)$ is slightly narrower than the exact solution. (Bottom) Corresponding frequency spectra.

where $q=\Gamma(3 / 4) / \Gamma(1 / 4) \approx 1 / 3$. This form for the $z$-dependent bunching envelope induced by the laser closely matches exact solutions, as shown in Figure 1. The rms width of $|g(z)|^{2}$ is, to a good approximation,

$$
\sigma_{g} \approx \frac{\sigma_{L}}{m^{1 / 3}}
$$

Thus we recover the $m^{-1 / 3}$ scaling of the harmonic compression effect of the initial laser pulse length [13, 14], where for large harmonics $\left(j_{m, 1}^{\prime}\right)^{2}-m^{2} \approx 1.62 m^{4 / 3}$. The FWHM has the same scaling but with a different proportionality, $\Delta_{g} \approx$ $\frac{1.4}{m^{1 / 3}} \Delta_{L}$, where $\Delta_{L}=\sigma_{L} \sqrt{8 \ln 2}$ is the FWHM of the seed laser intensity. These scalings are plotted against exact values in Figure 2.

Harmonic compression is the result of the high harmonics being increasingly more sensitive to the optimal modulation amplitude to produce bunching, so the longitudinal region of the Gaussian modulation that matches this condition becomes narrowed. The flattened, super-Gaussian form of $g(z)$ is thus characteristic of the optimized bunching envelope for a Gaussian second seed laser. We note that it slightly underestimates the exact longitudinal width of the bunching envelope, as shown in Figures 1, 2. The rms value in (13) is low by about $10 \%$, so the predicted spectral bandwidths can also differ slightly, but the scaling with $m$ still holds. 


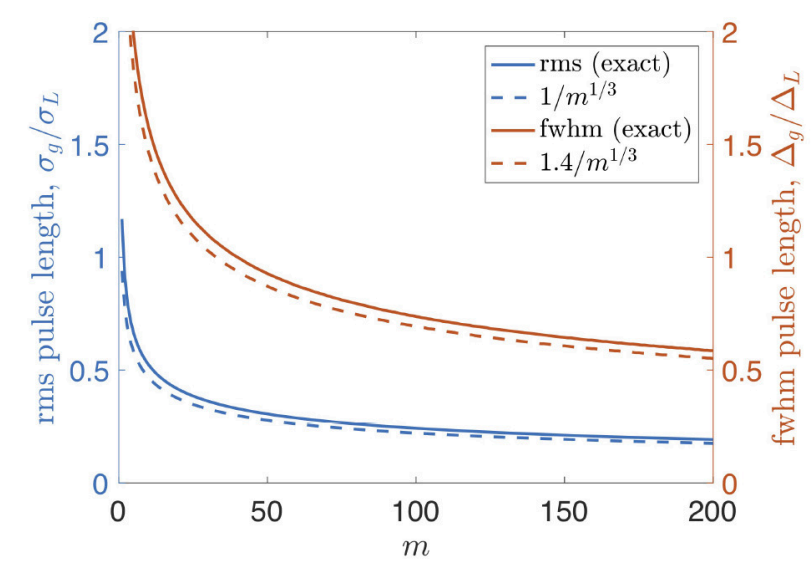

FIGURE 2 | Normalized RMS and FWHM lengths of the bunching envelope from exact numerical calculations (solid lines) and from the super-Gaussian approximation (dashed lines).

With an analytic form for $g(z)$ in hand, the transform-limited bandwidth in Equation (9) can be calculated for a specified electron beam distribution $f(z)$. For the sake of simplified calculations, we assume that $f(z)$ has the same, super-Gaussian form as $g(z)$, but with a different rms, $\sigma_{z}$, and properly normalized,

$$
f(z)=\frac{2 \sqrt{q}}{\sigma_{z} \Gamma(1 / 4)} \exp \left[-q^{2}\left(\frac{z}{\sigma_{z}}\right)^{4}\right] .
$$

The rms transform-limited bandwidth of the bunching spectrum then has simple analytic solution that is approximately (see Supplementary Material),

$$
\sigma_{k_{E}}^{2} \approx \frac{1}{2}\left(\frac{1}{\sigma_{z}^{4}}+\frac{1}{2 \sigma_{g}^{4}}\right)^{1 / 2}=\frac{1}{2}\left(\frac{1}{\sigma_{z}^{4}}+\frac{m^{4 / 3}}{2 \sigma_{L}^{4}}\right)^{1 / 2}
$$

Similarly, the rms length of the bunching envelope $\left|\tilde{b}_{n, m}(z)\right|^{2}$ is simply (see Equation 51),

$$
\sigma_{b} \approx \frac{1}{\sqrt{3} \sigma_{k_{E}}}
$$

Figure 3 shows how $\sigma_{k_{E}}$ varies with $\sigma_{L}$ for different harmonics. Two limiting regimes can be identified. If the laser is much longer than the beam $\sigma_{L} \gg m^{1 / 3} \sigma_{z}$, then $\sigma_{k_{E}}$ is independent of $m$ and is at its narrowest value. This is the regime studied in Hemsing [1]. In the opposite limit, with $g(z)$ much shorter than the electron beam, the bandwidth grows like $m^{1 / 3}$ because of the harmonic compression effect. Note that the relative bandwidth decreases in this limit like $m^{2 / 3}$,

$$
\sigma_{v_{E}}=\frac{\sigma_{k_{E}}}{k_{E}} \sim \frac{\sigma_{v_{L}}}{m^{2 / 3}}
$$

assuming $k_{E}=a_{E} k_{1} \approx m k_{2}$, and $\sigma_{v_{L}}=1 / 2 \sigma_{L} k_{2}$ is the relative bandwidth of the laser.

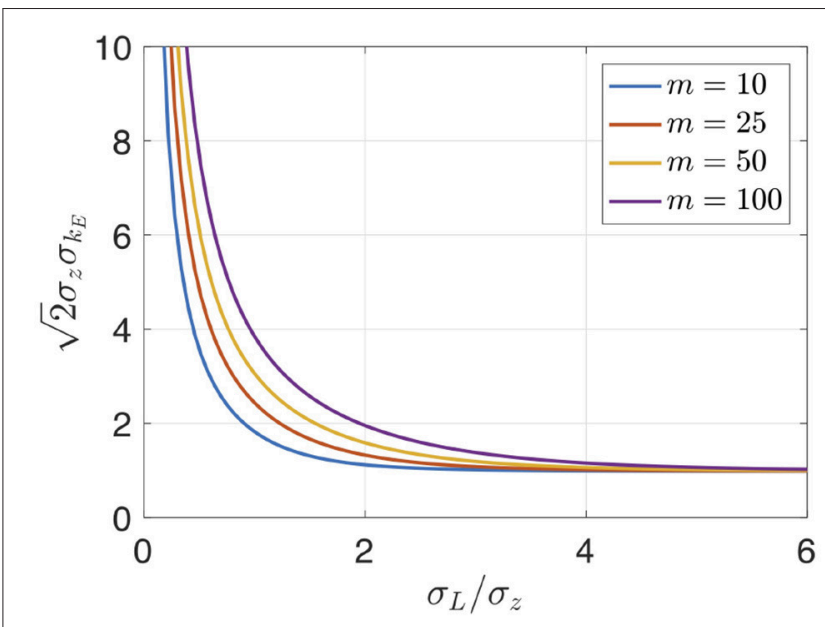

FIGURE 3 | Transform-limited bandwidth $\sigma_{k_{E}}$ as a function of laser pulse length $\sigma_{L}$ from Equation (15). Because of harmonic compression, the laser pulse length needs to increase slightly with increasing harmonic number to maintain a fixed bandwidth.

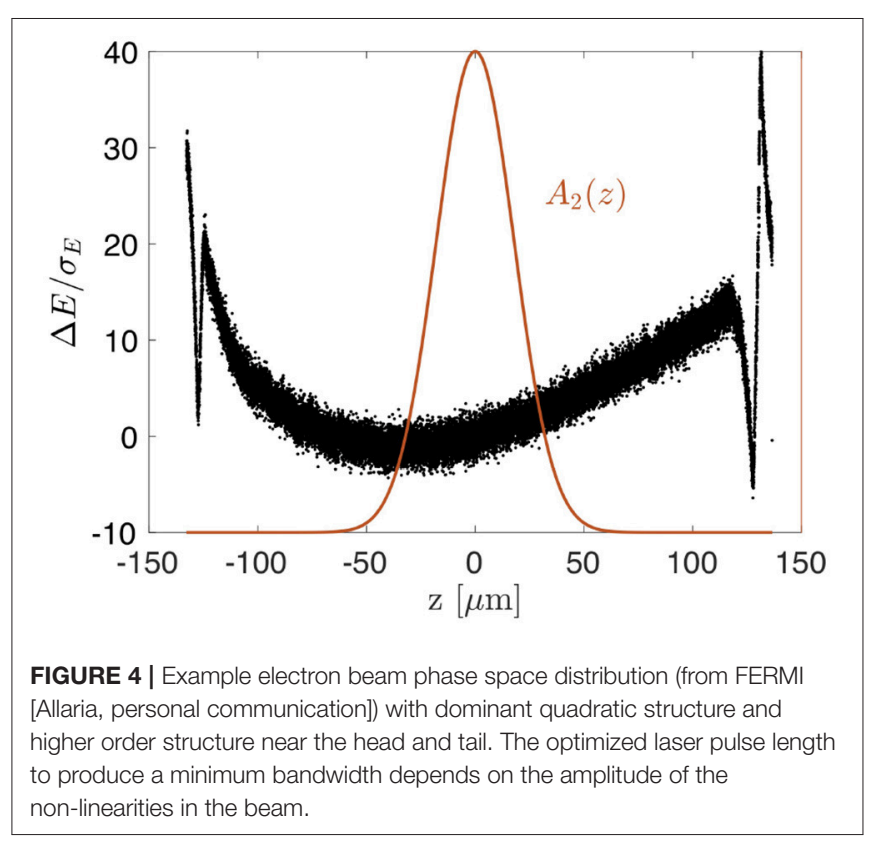

In general, the transform-limited bunching bandwidth $\sigma_{k_{E}}$ is set by the length of the laser on the electron beam. However, nonlinear phase structures $\varphi(z)$ introduce additional bandwidth through the term $\sigma_{\varphi^{\prime}}$ in Equation (8). Depending on the origin of the nonlinear phase structures, it may be possible to limit their impact on the total bandwidth by adjusting $\sigma_{k_{E}}$, either with the laser pulse length $\sigma_{L}$ or the electron beam length $\sigma_{z}$. In other words, what combination of $\sigma_{L}$ or $\sigma_{z}$ gives the smallest total bandwidth of the bunching spike $\sigma_{k}$ ? Consider the example of an electron beam with a quadratic chirp, as shown in Figure 4. On one hand, a narrowband laser with a longer pulse length could work, but it may extend over or add more of the undesirable nonlinearities that add bandwidth 
during the harmonic up-conversion. Conversely, a short laser pulse is intrinsically broadband, but may be less impacted by the presence of nonlinearities. As we will see, the optimization is straightforward once the phase is known, and the answer depends on the amplitude and source of the non-linearity.

\section{SIMPLE NONLINEAR STRUCTURE}

Without regard to the origin of the nonlinear phase structure (i.e., laser phase $\psi_{2}$ or electron beam energy structures $\Delta p_{1,2}$ ), let us first calculate the impact of generalized phase structures on the bunching bandwidth $\sigma_{k}$. We will then use the results to derive conditions for obtaining the minimum bandwidth.

Arbitrary continuous phase distortions can be expanded in a Taylor series about $z=0$. Similar to the temporal phase in laser physics, we can expand the bunching phase $\varphi(z)$ as,

$$
\varphi(z)=\sum_{N=1}^{\infty} \frac{\phi_{N}}{N !} z^{N}
$$

where each $\phi_{N}=\partial^{N} \varphi(z) /\left.\partial z^{N}\right|_{z=0}$ is real. The absolute phase of the pulse $\phi_{0}$ is ignored, as it does not affect the frequency or bandwidth. With Equation (6), the instantaneous frequency is $k_{z}(z)=k_{E}+\phi_{1}+\phi_{2} z+\frac{\phi_{3}}{2} z^{2}+\ldots$ Combined with (7) and (8), the bandwidth $\sigma_{\varphi^{\prime}}^{2}$ associated with the energy distortions can be calculated analytically up to arbitrary order in $N$ (see Supplementary Material).

\section{SINGLE PHASE TERM}

The analysis is simplified by isolating a single term in the phase expansion,

$$
\varphi(z)=\frac{\phi_{N}}{N !} z^{N}
$$

The total bunching bandwidth is then written in terms of the nonlinear coefficient $\phi_{N}$, as

$$
\sigma_{k}^{2}=\sigma_{k_{E}}^{2}\left(1+\frac{G_{N} \phi_{N}^{2}}{\sigma_{k_{E}}^{2 N}}\right) .
$$

The second term is the excess bandwidth from the phase nonlinearity. The analytic expression for the numerical coefficient $G_{N}$ is given in the Supplementary Material, and the lowest order numerical values are given in Table $\mathbf{1}$.

TABLE 1 | Numerical values of $G_{N}$.

\begin{tabular}{c|c}
$G_{1}$ & 0 \\
$G_{2}$ & 0.34 \\
$G_{3}$ & 0.035 \\
$G_{4}$ & 0.0073 \\
$G_{5}$ & 0.00046 \\
$G_{6}$ & 0.000033
\end{tabular}

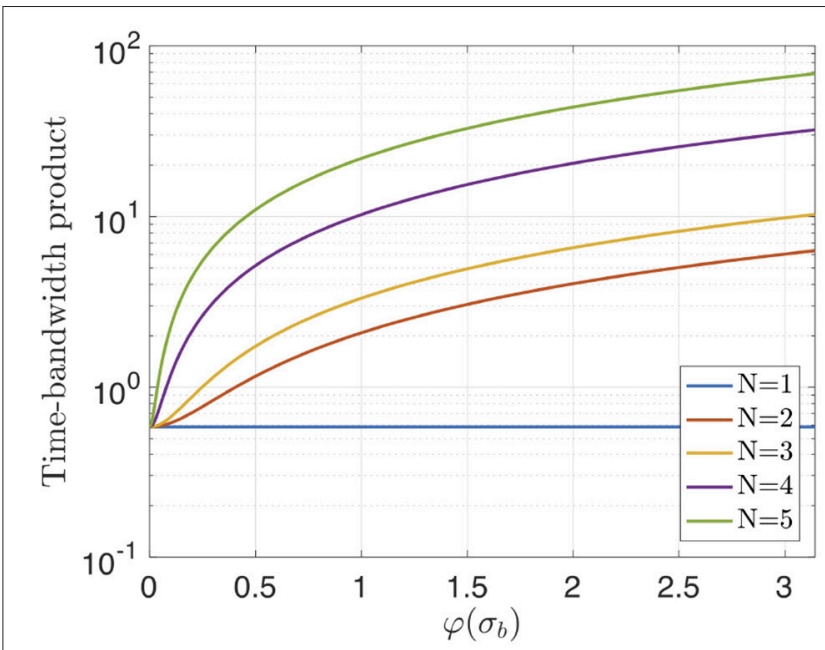

FIGURE 5 | Time-bandwidth product as a function of the phase change over the rms bunching envelope $\varphi\left(\sigma_{b}\right)=\phi_{N} \sigma_{b}^{N} / N$ !. Results are predicted from Equation (21)

The time-bandwidth product (TBP) of the bunching spectrum is the dimensionless product of the rms envelope length (16) and the rms bandwidth (20),

$$
T B P=\sigma_{b} \sigma_{k} \approx \frac{1}{\sqrt{3}} \sqrt{1+\frac{G_{N} \phi_{N}^{2}}{\sigma_{k_{E}}^{2 N}}} .
$$

Figure 5 plots shows how the TBP is impacted by the different order nonlinear phases. Here we see that larger order longitudinal phase structures have a larger effect on the TBP. This is in contrast to different order spectral phases in which only the even orders broaden the bunching bandwidth [14].

\section{Minimum Bandwidth}

With the expression for the bunching bandwidth in (20), it is straightforward to find the values of the laser pulse length or the electron beam length that minimize $\sigma_{k}$ in the presence of a phase nonlinearity. In either case this amounts to finding the optimal value of $\sigma_{k_{E}}$ for a given $\phi_{N}$. Assuming that $\phi_{N}$ is fixed with respect to the parameter being changed, the minimum bandwidth occurs when $\sigma_{k_{E}}$ can be adjusted to satisfy,

$$
\sigma_{k_{E}}^{2 N}=\phi_{N}^{2} G_{N}(N-1) .
$$

The minimum total bandwidth then scales directly with optimal $\sigma_{k_{E}}$,

$$
\left(\sigma_{k}\right)_{\min }=\sigma_{k_{E}} \sqrt{\frac{N}{N-1}} .
$$

An example of how the minimum bandwidth varies in general for different amplitude phase distortions is shown in Figure 6.

The full expressions for the optimal electron beam length and laser pulse length are given in (60) and 


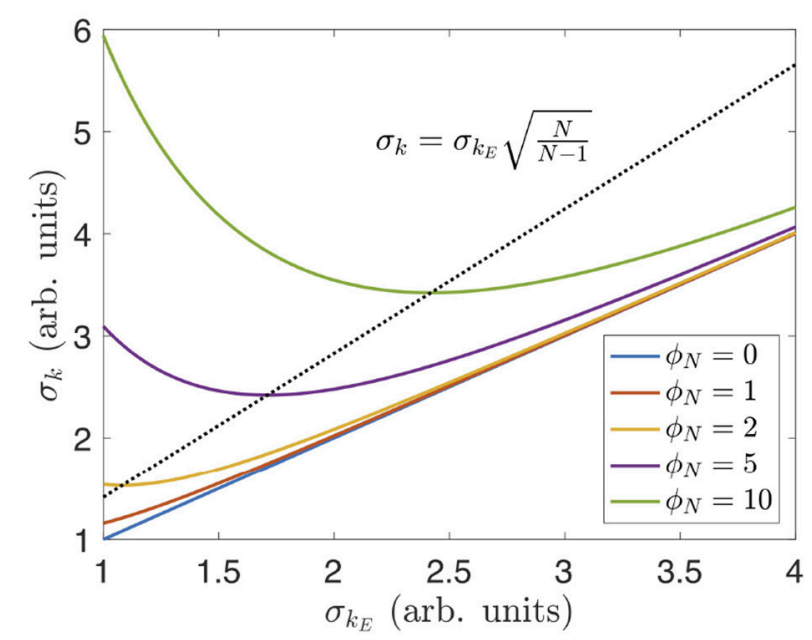

FIGURE 6 | Total bandwidth vs. $\sigma_{k_{E}}$ for different $N=2$ phase distortions. The minimum total bandwidth follows the dashed curve if $\phi_{N}$ is large enough (i.e., if Equation 22 is satisfied). Otherwise the minimum is set by the minimum possible value of $\sigma_{k_{E}}\left(\sigma_{k_{E}}=1\right.$, in this example for $\phi_{N}=0$ and 1).

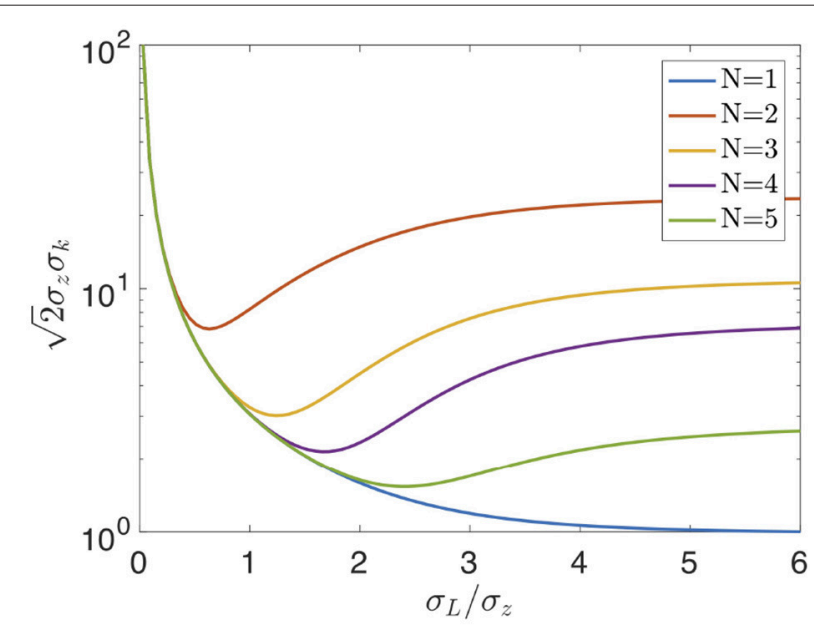

FIGURE 7 | Scaled bunching bandwidth vs. relative laser duration for phase distortions of $\phi_{N} \sigma_{Z}^{N}=20$ caused by different orders. Harmonic $m=50$ is modeled.

(62) the Supplementary Material. Consider the case where the transform-limited laser pulse length can be varied over a fixed length electron beam. If the phase distortion is large enough that $\phi_{N}^{2} G_{N}(N-1) \gg 1 /\left(2 \sigma_{z}^{2}\right)^{N}$, then the laser that minimizes the bandwidth is given by

$$
\left(\sigma_{L}^{2}\right)_{\min }=\frac{m^{2 / 3}}{2 \sqrt{2}\left[\phi_{N}^{2} G_{N}(N-1)\right]^{1 / N}} .
$$

Figure 7 illustrates this scenario. Identical values of the dimensionless phase distortion $\phi_{N} \sigma_{z}^{N}=20$ for different orders
$N$ show that, as $N$ is increased, shorter and shorter laser pulses are needed to minimize the bandwidth, as given by Equation (24).

Alternatively, if the laser is held fixed and $\phi_{N}^{2} G_{N}(N-1) \gg\left(m^{2 / 3} / \sqrt{8} \sigma_{L}^{2}\right)^{N}$, the optimal electron bunch length is

$$
\left(\sigma_{z}^{2}\right)_{\min }=\frac{1}{2\left[\phi_{N}^{2} G_{N}(N-1)\right]^{1 / N}} .
$$

Note that this is independent of the harmonic, in contrast to the optimal laser pulse length.

The TBP at the minimum bandwidth is,

$$
T B P=\frac{1}{\sqrt{3}} \sqrt{\frac{N}{N-1}} .
$$

It has the same dependence on the order of the phase distortion as $\sigma_{k}$; both approach their transform-limited values as $N$ becomes large. Clearly the lowest order phase distortions have the greatest impact on both. These results shows that, if the phase distortions are described by a single polynomial term, then by proper optimization the TBP and the bandwidth will be no more than $\sqrt{2}$ times the transform-limit.

Inspection of the full expression for the TBP shows that it cannot be minimized simultaneously with the total bandwidth by adjustment of $\sigma_{k_{E}}$ alone, though it is possible if $\phi_{N}$ is not held fixed.

\section{Quadratic Electron Beam Chirp}

In modern FELs, it is common that the electron beam has some residual energy-time correlation in the phase space. These correlations can be the result of wakefields or other collective effects during compression and transport. They may be difficult to remove completely, and may be present at the entrance to the EEHG seeding system, or can develop within the EEHG beam line from collective effects [1]. In either case, their impact on the final bunching bandwidth can be calculated within the present framework and stated constraints.

Consider a purely quadratic energy chirp on the electron beam of the form,

$$
\Delta p(z)=h_{2} k_{2}^{2} z^{2} .
$$

The chirp amplitude is characterized by the dimensionless factor $h_{2}$. Assuming the second seed laser is transform-limited $\left(\psi_{2}=\right.$ $0)$, the bunching phase can be written generically as [1],

$$
\varphi(z)=-\eta \Delta p(z)
$$

where $\eta=\xi_{E}$ or $a_{E} B_{2}$ depending on where the energy distortion occurs, as expressed in Equation (3). The nonlinear coefficient in (19) for $N=2$ is then

$$
\phi_{2}=-2 \eta h_{2} k_{2}^{2}
$$

Plugging this into Equation (22) and optimizing the laser pulse length, one can show that if the amplitude of the quadratic beam chirp strongly satisfies

$$
h_{2}>\frac{\sqrt{3}}{4 \eta\left(k_{2} \sigma_{z}\right)^{2}},
$$


then the laser pulse length that minimizes the bandwidth is, from Equation (24),

$$
\left(\sigma_{L}\right)_{\min } \approx \frac{5 m^{1 / 3}}{9 k_{2} \sqrt{\eta h_{2}}} .
$$

The corresponding relative bandwidth from Equation (23) is then

$$
\left(\sigma_{\nu}\right)_{\min }=\frac{\left(\sigma_{k}\right)_{\min }}{k_{E}} \approx \frac{3 \sqrt{\eta h_{2}}}{2 m} .
$$

Consider the realistic case of electron beams at the FERMI FEL [23] (for example in Figure 4), which can have a quadratic chirp of $30 \mathrm{MeV} / \mathrm{ps}^{2}$ upstream of the $260 \mathrm{~nm}$ seeding sections. This corresponds to $h_{2}=2 \times 10^{-6}$ for a slice energy spread of $\sigma_{E}=0.15 \mathrm{MeV}$. In this case Equation (30) is satisfied for beams longer than $\sigma_{z}=30 \mu \mathrm{m}$ (100 fs), which is easily the case for the ps-scale beams at FERMI (assuming $\eta=1 / 2$ ). The minimum bandwidth setting from (31) requires a laser pulse duration of $250 \mathrm{fs}$ at the 36th harmonic and produces $\left(\sigma_{v}\right)_{\min }=4.2 \times 10^{-5}$. At the 64th harmonic, a 300 fs laser is optimum to generate $\left(\sigma_{v}\right)_{\min }=2.3 \times 10^{-5}$

\section{Linear Laser Frequency Chirp}

Similar to quadratic structure on the electron beam, a linear laser chirp in the second seed laser introduces frequency-time correlations that mix into the harmonic up-conversion process and impact the final bunching bandwidth. If the electron beam phase space is flat, the bunching phase $\varphi(z)$ in Equation (3) is only the laser phase times the harmonic,

$$
\varphi(z)=m \psi_{2}(z)
$$

It is useful to separate laser chirps according to whether they are defined in terms of a fixed pulse length as in Siegman [24] or in terms of a fixed bandwidth (e.g., [25]).

\section{Fixed Laser Pulse Length}

A linear frequency chirp corresponds to a quadratic phase of the form [24],

$$
\psi_{2}(z)=\alpha z^{2}
$$

Assuming the pulse length is fixed, $\alpha$ only affects the laser bandwidth. The quadratic coefficient of the bunching phase is then $\phi_{2}=2 m \alpha$, and the analysis is the same as with a quadratic e-beam chirp with the replacement $-\eta h_{2} k_{2}^{2}=m \alpha$. If both the e-beam and the laser phase have quadratic structure, then their effect on the bunching cancels if $\eta h_{2} k_{2}^{2}=m \alpha$.

\section{Fixed Laser Bandwidth}

On the other hand, for a fixed-bandwidth laser pulse with rms intensity $\sigma_{k_{L}}$, a linear frequency chirp that changes only the pulse length $\sigma_{L}$ corresponds to a phase,

$$
\psi_{2}(z)=C \sigma_{k_{L}}^{2} z^{2}
$$

where the chirp is

$$
C= \pm \frac{\sqrt{x^{2}-1}}{x^{2}}
$$

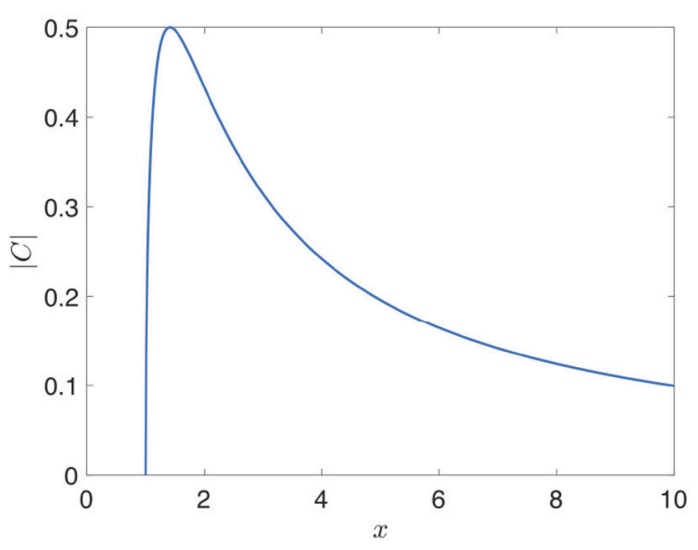

FIGURE $8 \mid$ Chirp as a function of stretch factor, $x=\sigma_{L} / \sigma_{L_{0}}$. From Equation (36).

and

$$
x=\frac{\sigma_{L}}{\sigma_{L_{0}}}=2 \sigma_{L} \sigma_{k_{L}} \geq 1,
$$

is the pulse stretch factor. The laser bandwidth corresponds to transform-limited pulse length, $\sigma_{L_{0}}=1 / 2 \sigma_{k_{L}}$.

Note that the chirp grows from zero to a maximum of $|C|=$ $1 / 2$ when the pulse length is stretched by $x=\sqrt{2}$, and then decreases to zero as the pulse is stretched further. This is shown in Figure 8. This means that a strongly stretched pulse can have the same small chirp as a barely stretched pulse, even though the pulse lengths are quite different [25]. Wigner distributions of the laser pulses for different values of $x$ are shown in Figure 9.

The quadratic coefficient of the bunching phase is,

$$
\phi_{2}=2 m C \sigma_{k_{L}}^{2} \text {. }
$$

The chirp is multiplied by the harmonic. Because the laser pulse length changes, the bandwidth $\sigma_{k_{E}}$ is also a function of the stretch factor $x$,

$$
\sigma_{k_{E}}^{2}=\frac{1}{2}\left(\frac{1}{\sigma_{z}^{4}}+\frac{8 m^{4 / 3} \sigma_{k_{L}}^{4}}{x^{4}}\right)^{1 / 2} .
$$

Inserting (38) into (22), one can see that if the pulse is stretched such that $\frac{2}{3} m^{2 / 3}\left(x^{2}-1\right)>1$, then there exists a finite length electron beam that minimizes the bandwidth, given approximately by (25),

$$
\left(\sigma_{z}\right)_{\min } \approx \frac{3^{1 / 4}}{2 \sigma_{k_{L}} \sqrt{m C}} .
$$

The optimum electron beam length is the smallest at the maximum chirp, $C=1 / 2$, and is also less than $\sigma_{L_{0}}$ unless the harmonic chirp is smaller than one, $m C<1$. This can occur for both barely stretched or strongly stretched pulses. The minimum relative bandwidth from Equation (23) is then,

$$
\left(\sigma_{v}\right)_{\min }=\frac{2 \sigma_{v_{L}}}{3^{1 / 4}} \sqrt{\frac{C}{m}} .
$$

Figure 10 shows how the bandwidths evolve for different $\sigma_{z}$. 

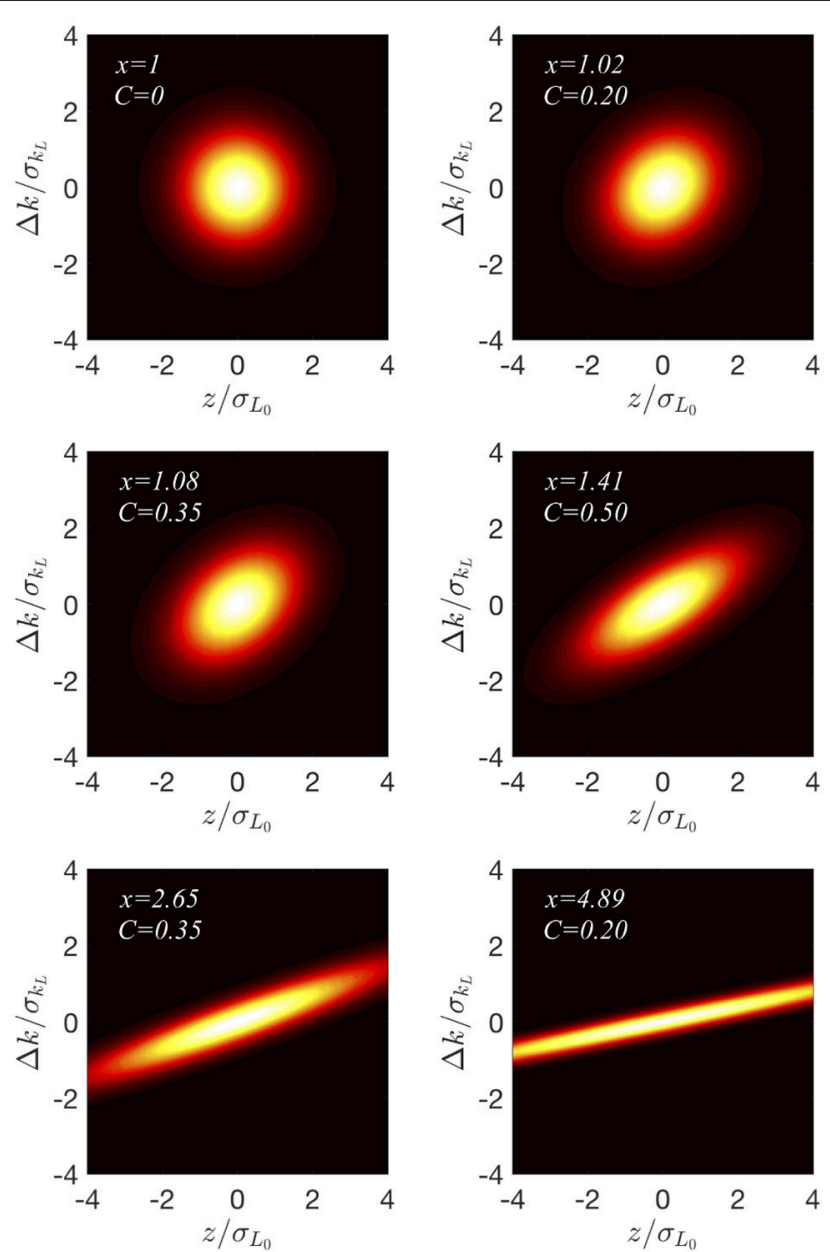

FIGURE 9 | Wigner distributions of a chirped laser pulse with fixed bandwidth with $x$ and $C$ values from Equation (36).

Note that if the electron beam also has quadratic curvature, the laser chirp can be set to negate the bandwidth broadening if $C=-\eta h_{2} / m \sigma_{v_{L}}^{2}$, similar to [4]. Consider the example of the 4 $\mathrm{GeV}\left(\sigma_{E}=0.45 \mathrm{MeV}\right)$ beam at LCLS-II using EEHG to reach harmonic $m=130$ of a $260 \mathrm{~nm}$ laser. Assuming $\sigma_{z}=15 \mu \mathrm{m}$ (50 fs), and quadratic electron beam curvature $h_{2}=1 \times 10^{-4}$ in the beam core, the transform-limited laser pulse to minimize the bandwidth from (30) is also $50 \mathrm{fs}$ (assuming $\eta=1 / 2$ ). This yields a relative bandwidth from $(32)$ of $\left(\sigma_{v}\right)_{\min } \approx 8 \times 10^{-5}$. However, chirping the laser slightly $C=-\eta h_{2} / m \sigma_{v_{L}}^{2} \approx-1 / 4$, (stretching the pulse by about $3 \%$ ), the bandwidth is reduced by $\sqrt{2}$ and is at the transform limit.

Finally, it is useful to look at a practical example in which the electron beam is long compared to the length of the second laser, irrespective of the chirp (e.g., FERMI). The total relative bandwidth from Equation (20) is then

$$
\sigma_{v}^{2} \approx \frac{4 \sigma_{v_{L}}^{2}}{3 x^{2} m^{4 / 3}}\left[1+\frac{3}{4} m^{2 / 3}\left(x^{2}-1\right)\right]
$$

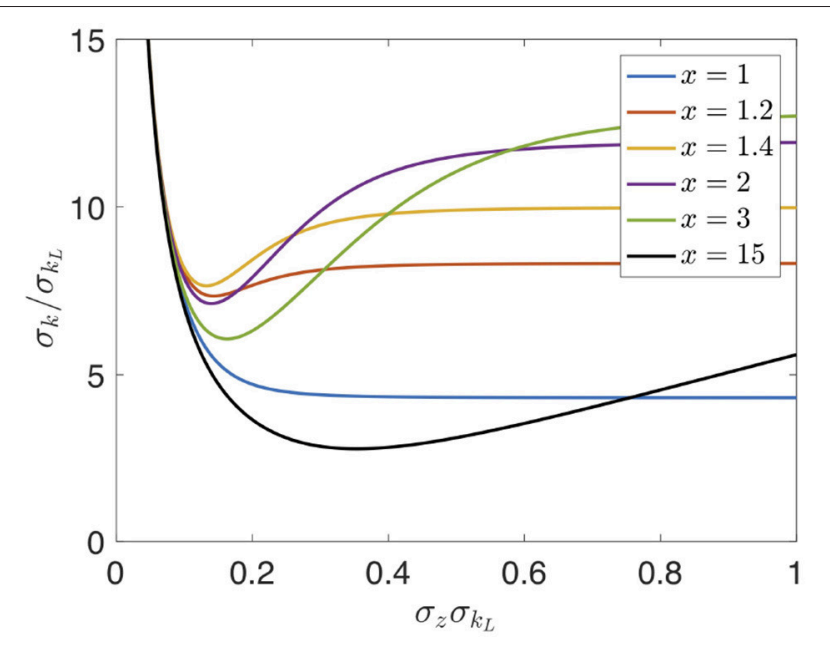

FIGURE 10 | Scaled bandwidth vs. electron beam length for different values of a fixed-badwidth laser stretched with a linear chirp at $m=50$.

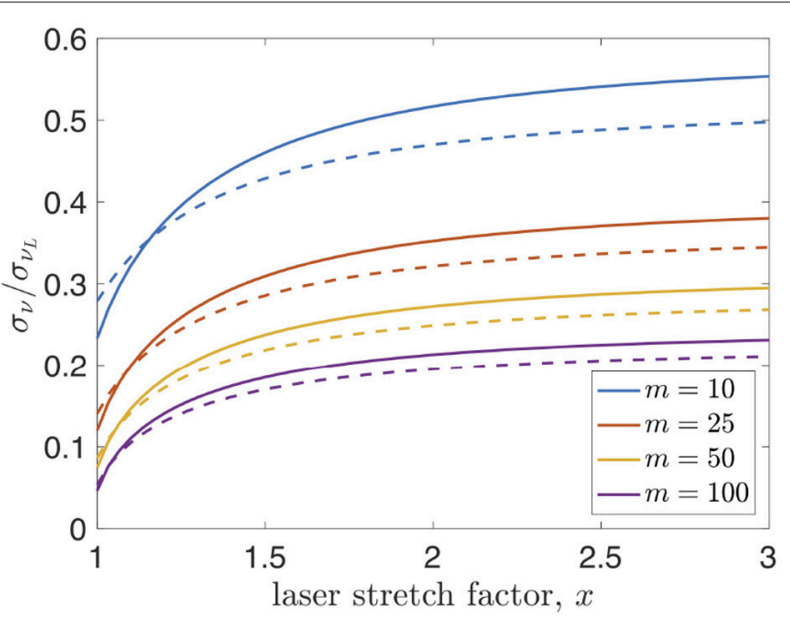

FIGURE 11 | Relative bandwidth as the fixed-bandwidth second laser is stretched for different harmonics, assuming $\sigma_{z} \rightarrow \infty$. Exact solutions are solid lines, and approximate solutions from Equation (42) are dashed lines. The disagreement with exact solutions is attributed to the super-Gaussian approximation for $g(z)$.

Inspection reveals that $\sigma_{v}$ grows with increasing $x$ for high harmonics, so the minimum bandwidth is obtained only when the laser chirp is zero. The scaling with the harmonic number clearly differs according to whether the laser is fully compressed or stretched,

$$
\sigma_{v} \approx \begin{cases}\sigma_{v_{L}} / m^{2 / 3} & (x=1) \\ \sigma_{v_{L}} / m^{1 / 3} & (x \gg 1)\end{cases}
$$

Figure 11 illustrates the bandwidth growth between the two regimes. 


\section{CONCLUSIONS}

Finite laser pulse length effects in EEHG FEL seeding are investigated. We have derived an approximate super-Gaussian form for the laser modulation $g(z)$ and used it to calculate the spectral bandwidth of the harmonic bunching peak. The effects of nonlinear longitudinal variations in the ebeam energy and the laser temporal phase are studied by way of a general phase term $\varphi(z)$. From the series expansion, the impact on the bandwidth and the TBP of the different order phase contributions is determined. All orders of the temporal phase contribute, in direct contrast to the spectral phase where the odd orders are negligible. Conditions on obtaining the minimum bandwidth are derived, which is shown to exceed the transform-limited bandwidth by at most $\sqrt{2}$, depending on the order of the nonlinearity.

\section{REFERENCES}

1. Hemsing E. Bunching phase and constraints on echo enabled harmonic generation. Phys Rev Accel Beams. (2018) 21:050702. doi: 10.1103/PhysRevAccelBeams.21.050702

2. Allaria E, Appio R, Badano L, Barletta W, Bas-sanese S, Biedron S, et al. Highly coherent and stable pulses from the FERMI seeded free-electron laser in the extreme ultraviolet. Nat Photon. (2012) 6:699. doi: 10.1038/nphoton. 2012.233

3. Allaria E, Castronovo D, Cinquegrana P, Craievich P, Dal Forno M, Danailov M, et al. Two-stage seeded soft-X-ray free-electron laser. Nat Photon. (2013) 7:913. doi: 10.1038/nphoton.2013.277

4. Gauthier D, Ribič PCVR, De Ninno G, Allaria E, Cinquegrana P, Danailov $\mathrm{MB}$, et al. Spectrotemporal shaping of seeded free-electron laser pulses. Phys Rev Lett. (2015) 115:114801. doi: 10.1103/PhysRevLett.115.114801

5. Labat M, Joly N, Bielawski S, Szwaj C, Bruni C, Couprie ME. Pulse splitting in short wavelength seeded free electron lasers. Phys Rev Lett. (2009) 103:264801. doi: 10.1103/PhysRevLett.103.264801

6. Gauthier D, Ribič PCVR, De Ninno G, Allaria E, Cinquegrana P, Danailov MB, et al. Generation of phase-locked pulses from a seeded free-electron laser. Phys Rev Lett. (2016) 116:024801. doi: 10.1103/PhysRevLett.116.024801

7. Petralia A, Anania MP, Artioli M, Bacci A, Bellaveglia M, Carpanese $\mathrm{M}$, et al. Two-color radiation generated in a seeded free-electron laser with two electron beams. Phys Rev Lett. (2015) 115:014801. doi: 10.1103/PhysRevLett.115.014801

8. Roussel E, Ferrari E, Allaria E, Penco G, Di Mitri S, Veronese $\mathrm{M}$, et al. Multicolor high-gain free-electron laser driven by seeded microbunching instability. Phys Rev Lett. (2015) 115:214801. doi: 10.1103/PhysRevLett.115.214801

9. Hemsing E, Stupakov G, Xiang D, Zholents A. Beam by design: laser manipulation of electrons in modern accelerators. Rev Mod Phys. (2014) 86:897. doi: 10.1103/RevModPhys.86.897

10. Stupakov G. Using the beam-echo effect for generation of short-wavelength radiation. Phys Rev Lett. (2009) 102:074801. doi: 10.1103/PhysRevLett.102.074801

11. Xiang D, Stupakov G. Echo-enabled harmonic generation free electron laser. Phys Rev ST Accel Beams. (2009) 12:030702. doi: 10.1103/PhysRevSTAB.12.030702

12. Huang Z, Ratner D, Stupakov G, Xiang D. Effects of energy chirp on echoenabled harmonic generation free-electron lasers. In: Proceedings of the 2009 Free-Electron Laser Conference. Liverpool (2009). p. 127.

13. Stupakov G. SLAC-PUB-14639. Effect of finite pulse length and laser frequency chirp on HGHG and EEHG seeding. SLAC-PUB-14639. (2011)

\section{AUTHOR CONTRIBUTIONS}

The author confirms being the sole contributor of this work and has approved it for publication.

\section{ACKNOWLEDGMENTS}

This work was supported by the U.S. Department of Energy Contract No. DE-AC02-76SF00515 and the U.S. DOE Office of Basic Energy Sciences under award number 2017-SLAC100382. The author would like to thank L. Giannessi for helpful discussions.

\section{SUPPLEMENTARY MATERIAL}

The Supplementary Material for this article can be found online at: https://www.frontiersin.org/articles/10.3389/fphy. 2019.00035/full\#supplementary-material

14. Ratner D, Fry A, Stupakov G, White W. Laser phase errors in seeded free electron lasers. Phys Rev ST Accel Beams. (2012) 15:030702. doi: 10.1103/PhysRevSTAB.15.030702

15. Geloni G, Kocharyan V, Saldin E. Analytical studies of constraints on the performance for eehg fel seed lasers (2011). arXiv:1111.1615.

16. Penn G. Tech. Rep. NGLS Technical Note 35, LBNL (2012).

17. Zhao ZT, Wang D, Chen JH, Chen ZH, Deng HX, Ding JG, et al. First lasing of an echo-enabled harmonic generation free-electron laser. Nat Photon. (2012) 6:360. doi: 10.1038/nphoton.2012.105

18. Penn G. Stable, coherent free-electron laser pulses using echo-enabled harmonic generation. Phys Rev ST Accel Beams. (2014) 17:110707. doi: 10.1103/PhysRevSTAB.17.110707

19. Rebernik Ribič P, Roussel E, Penn G, De Ninno G, Giannessi L, Penco G, et al. Echo-enabled harmonic generation studies for the FERMI free-electron laser. Photonics. (2017) 4:19. doi: 10.3390/photonics4010019

20. Hemsing E, Garcia B, Huang Z, Raubenheimer T, Xiang D. Sensitivity of echo enabled harmonic generation to sinusoidal electron beam energy structure. Phys Rev Accel Beams. (2017) 20:060702. doi: 10.1103/PhysRevAccelBeams.20.060702

21. Penn G, Garcia B, Hemsing E, Marcus G. Constraints on pulse duration produced by echo-enabled harmonic generation. In: Proceedings, 38th International Free Electron Laser Conference, FEL2017. Santa Fe, NM (2018). p. MOP010.

22. Lilly JM, Olhede SC. Bivariate Instantaneous Frequency and Bandwidth. IEEE Trans Signal Process. (2010) 58:591. doi: 10.1109/TSP.2009.2031729

23. Giannessi L, Masciovecchio C. FERMI: present and future challenges. Appl Sci. (2017) 7:640. doi: 10.3390/app7060640

24. Siegman A. Lasers. Sausalito, CA: University Science Books (1986).

25. Huang S, Ding Y, Feng Y, Hemsing E, Huang Z, Krzywinski J, et al. Generating single-spike hard X-ray pulses with nonlinear bunch compression in free-electron lasers. Phys Rev Lett. (2017) 119:154801. doi: 10.1103/PhysRevLett.119.154801

Conflict of Interest Statement: The author declares that the research was conducted in the absence of any commercial or financial relationships that could be construed as a potential conflict of interest.

Copyright (c) 2019 Hemsing. This is an open-access article distributed under the terms of the Creative Commons Attribution License (CC BY). The use, distribution or reproduction in other forums is permitted, provided the original author(s) and the copyright owner(s) are credited and that the original publication in this journal is cited, in accordance with accepted academic practice. No use, distribution or reproduction is permitted which does not comply with these terms. 